\title{
Comportamiento poscosecha de frutos de ciruela (Prunus salicina Lindl.) en cuatro estados de madurez tratados con etileno
}

\section{Effect of ethylene on postharvest behavior of plum fruits (Prunus salicina Lindl.) at four stages of maturity}

JAVIER GIOVANNI ÁLVAREZ-HERRERA ${ }^{1,2}$

XIMENA ROZO-ROMERO' 1

ANDREA JOHANA REYES ${ }^{1}$

Estados de madurez en ciruela 'Horvin'.

Foto: X. Rozo-Romero

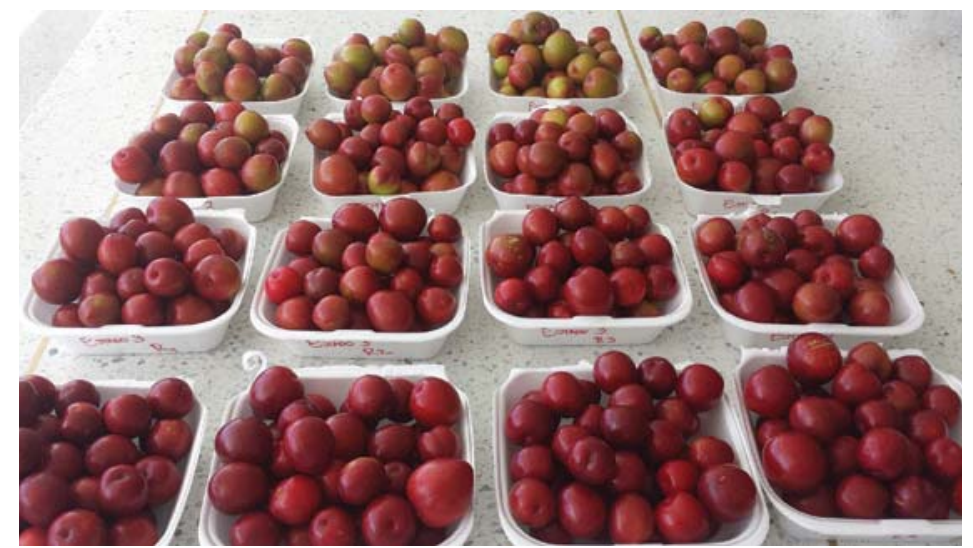

\section{RESUMEN}

La aplicación de etileno en frutos es comúnmente utilizada para uniformizar el color y acelerar la maduración, de esta manera se puede mejorar la apariencia y las características organolépticas. Por lo anterior, se trataron frutos de ciruela variedad Horvin recolectados en cuatro estados de madurez (2, 3, 4 y 5), con etileno comercialmente conocido como Ethrel en dosis de $100 \mu \mathrm{L} \mathrm{L}^{-1}$. Se utilizó un diseño completamente al azar con cuatro tratamientos (correspondientes a los estados de madurez) cada uno con 4 repeticiones, para un total de 16 unidades experimentales compuestas cada una por $400 \mathrm{~g}$ de frutos aproximadamente. Cada tres días se midió el color, pérdida de peso, tasa respiratoria, firmeza, $\mathrm{pH}$, acidez total titulable (ATT) y sólidos solubles totales (SST). Se realizaron mediciones hasta que los frutos perdieron calidad organoléptica. En las variables pérdida de peso, firmeza, SST, ATT y pH no hubo diferencia significativa en la mayoría de los puntos de muestreo. En cuanto al color hubo diferencias estadísticas. La tasa de respiración y la relación de madurez al final de las mediciones presentaron diferencias significativas, obteniendo los mayores valores en el estado de madurez 5 y los menores valores en los estados 3 y 2 respectivamente. Por lo tanto, el uso de etileno en frutos de ciruela es benéfico ya que favorece el cambio de color y aumenta la relación de madurez, por lo que garantiza en almacenamiento uniformidad entre estados de madurez.

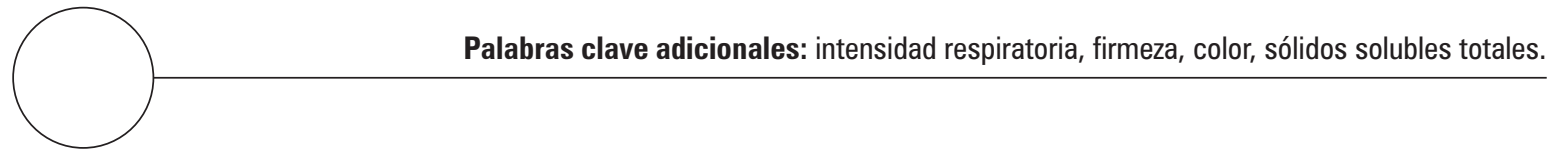

\footnotetext{
Facultad de Ciencias Agropecuarias, Grupo de Investigaciones Agrícolas, Universidad Pedagógica y Tecnológica de Colombia (UPTC), Tunja (Colombia).

2 Autor para correspondencia.jgalvarezh@gmail.com
} 


\section{ABSTRACT}

The application of ethylene is commonly used on fruits to standardize the color and accelerate ripening, which improves the appearance and organoleptic characteristics. Therefore, Horvin variety plum fruits were collected in four maturity stages (2, 3, 4 and 5) and treated with ethylene, commercially known as ethrel, in doses of $100 \mu \mathrm{L} \mathrm{L}^{-1}$. The experimental design was completely randomized with four treatments (corresponding to the states of maturity), with 4 replicates each, for a total of 16 experimental units, each consisting of approximately $400 \mathrm{~g}$ of fruit. Every three days, the variables of color, weight loss, respiratory intensity, firmness, $\mathrm{pH}$, total titratable acidity (TTA) and total soluble solids (TSS) were measured. The measurements were taken until the fruits lost organoleptic quality. For the variable of weight loss, firmness, TSS, pH and TTA, there were no significant differences in most of the sampling points between the treatments. However, there were significant differences for color between the treatments. As for the respiration intensity and maturity index at the end of the measurements, significant differences were observed, obtaining the highest values in maturity stage 5 and lower values in stages 3 and 2 . Therefore, the use of ethylene on plum fruits is beneficial because it favors a change in color and the maturity ratio increases, thus assuring uniformity in storage between the stages of maturity.

Additional key words: respiratory intensity, firmness, color, total soluble solids.

Fecha de recepción: 26-03-2015

Aprobado para publicación: 28-05-2015

\section{INTRODUCCIÓN}

Las ciruelas son frutos climatéricos caracterizados por tener la capacidad de continuar el proceso de maduración después de ser cosechados, debido a que presentan un rápido incremento en la actividad respiratoria y su producción de etileno continua después de la cosecha (Kays, 2004). Este proceso ocurre en varias fases, la primera se denomina madurez fisiológica y se refiere a la etapa en la cual el fruto ha tomado todos los nutrientes de la planta y ha alcanzado la capacidad de reproducirse, alcanzando la plenitud de su crecimiento, posteriormente en la segunda fase, denominada madurez de consumo el fruto evoluciona hasta adquirir la máxima calidad comestible y estética y finalmente se produce la etapa de envejecimiento o senescencia (Kader, 2002).

En frutos altamente perecederos como la ciruela, el conocimiento del momento óptimo para la cosecha es muy importante ya que el consumidor requiere de productos con una calidad comercial excelente, por lo que es necesa- rio utilizar técnicas que faciliten al productor entregar un fruto maduro comercialmente, lo que muchas veces se dificulta, pues la cosecha generalmente se realiza en un estado inmaduro cuando el fruto no ha alcanzado el color ni el sabor deseado, y no en el estado de madurez óptimo, como lo describen Tapia y Reginato (2012). Una alternativa para obtener en poco tiempo las características necesarias en el momento de la comercialización es la aplicación de etileno, ya que según afirma Kader (2002), este tratamiento ayuda a que los frutos maduren rápidamente para ser expuestos en anaquel, obteniendo pérdida de acidez y de astringencia, además de uniformizar el color del fruto.

El color es uno de los parámetros que más cambia durante la maduración, pues es el índice de madurez más utilizado en la cosecha y comercialización del fruto de ciruela (Verma y Joshi, 2000). Teniendo en cuenta este aspecto, el etileno es el responsable de acelerar los cambios de 
color relacionados con la producción de pigmentos antocianos y la pérdida de clorofila así como de incrementar la producción de aromas en los frutos (Montalvo-Gonzáles et al., 2009). La cianidin-3-galactosidasa es la principal antocianina responsable de la coloración de los frutos de ciruela durante la maduración (Paliyath y Murr, 2008); por tanto, un rápido desarrollo del color característico puede producir una calidad superior en el fruto.

Montalvo-González (2009) reporta que el etileno exógeno aumenta la velocidad de respiración y producción de etileno de los chiles verdes; pero no se desverdizaron. La aplicación de $1.000 \mu \mathrm{L} \mathrm{L}^{-1}$ de etileno exógeno en los frutos cosechados de color verde con áreas rojas $\leq 50 \%$ originó $100 \%$ de los frutos rojos, a los dos días después de la aplicación de etileno. En los frutos verdes, cuando se aplicó $500 \mu \mathrm{L} \mathrm{L}^{-1}$ a los $9 \mathrm{~d}$ de almacenamiento, se obtuvo el $20 \%$ de frutos rojos. En frutos de papaya 'Maradol' (Carica papaya L.), los resultados obtenidos por De la Cruz et al. (2009) indicaron que el primer cambio notable fue el color en la epidermis (amarillo) y en la pulpa (naranja), típicos en un fruto listo para el consumo. Los frutos expuestos a $500 \mu \mathrm{L} \mathrm{L}^{-1}$ de etileno por 16 y $24 \mathrm{~h}$ mostraron un pico climatérico un día después del tratamiento, mientras que los frutos expuestos por solamente $8 \mathrm{~h}$ mostraron su pico climatérico $2 \mathrm{~d}$ después de la exposición al etileno. La firmeza del fruto

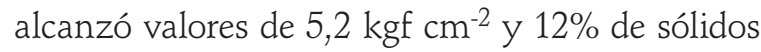
solubles a los $6 \mathrm{~d}$ a $25^{\circ} \mathrm{C}$. Los resultados sugieren que es posible acelerar la maduración de papaya 'Maradol' de 8 a 3 d por exposición a atmósferas con etileno. En frutos de pitahaya Deaquiz et al. (2014) mencionan que los frutos control y los tratados con etileno tendieron a un comportamiento respiratorio climatérico. El contenido en los carotenoides totales en los frutos fue significativamente mayor para la aplicación de etileno que para el tratamiento control lo que es consistente con el cambio en la coloración de los frutos.

Por tanto, el objetivo de esta investigación fue determinar el comportamiento poscosecha de los frutos de ciruela en cuatro estados de madurez y sometidos a etileno.

\section{MATERIALES Y MÉTODOS}

El trabajo se realizó en el laboratorio de Fisiología Vegetal de la Universidad Pedagógica y Tecnológica de Colombia, sede Tunja, los frutos utilizados fueron recolectados de un cultivo comercial en el municipio de Tuta (Boyacá) ubicado a 2.600 msnm, 0541'36" de latitud norte y longitud oeste $73^{\circ} 13^{\prime} 51^{\prime \prime}$, el cual presenta una precipitación promedio de $935 \mathrm{~mm}$ y una temperatura media que oscila entre los 12 y $14^{\circ} \mathrm{C}$.

Como material vegetal se utilizaron frutos de ciruela (Prunus domestica Lindl.) variedad Horvin. Los frutos fueron recolectados en cuatro estados de madurez (2, 3, 4 y 5) según la clasificación reportada por Sánchez y Saavedra (2013). Los frutos se escogieron de un tamaño homogéneo, libre de daños mecánicos y en buenas condiciones fitosanitarias. Se utilizó etefon como fuente de etileno (Ethrel® 48 SL, Bayer CropScience).

Se utilizó un diseño completamente al azar con 4 tratamientos (estados de madurez 2, 3, 4 y 5) cada uno con cuatro repeticiones, para un total de 16 unidades experimentales (UE). Cada UE estuvo compuesta por $400 \mathrm{~g}$ de frutos aproximadamente.

Se utilizaron frutos en estados de madurez 2 (75\% verde - $25 \%$ rojo), 3 (50\% verde - $50 \%$ rojo), 4 (25\% verde - $75 \%$ rojo) y 5 (100\% rojo), los cuales fueron desinfectados con una solución de hipoclorito de sodio al $1 \%$ y posteriormente se dejaron secar al aire durante 30 minutos. Se realizó la inmersión en etileno en dosis de $100 \mu \mathrm{L}$ $\mathrm{L}^{-1}$ durante $15 \mathrm{~min}$. Los frutos se empacaron en recipientes de Icopor, con capacidad de $450 \mathrm{~g}$ de fruto y se dejaron a temperatura ambiente.

Se realizaron mediciones hasta que los frutos perdieron la calidad organoléptica. En total se 
realizaron cuatro mediciones. Las variables medidas fueron las siguientes: la firmeza del fruto se determinó mediante la utilización de un penetrómetro digital PCE-PTR200 (PCE-Ibérica SL, Albacete, España) con aproximación 0,05 N. La pérdida de masa se determinó mediante la medición de la masa fresca en una balanza electrónica Acculab VIC 612 de 0,01g de precisión (Sartorius Spain S.A., Madrid) durante la fase poscosecha. Para esta variable se marcó un fruto por cada unidad experimental al cual se le realizaban las mediciones a través del tiempo. El color de la epidermis se midió mediante colorímetro digital marca Minolta CR 300 (Minolta Co., Tokyo) en el cual se determinaron los parámetros del sistema CIELab "L", "a" y "b", a cada fruto se le hicieron tres lecturas en el diámetro ecuatorial. L indica la luminosidad, donde 0 es negro y 100 blanco; valores de "a" $<0$ indican tendencia hacia el verde $y>0$ hacia el rojo; " $b$ " tiene el mismo rango pero valores $<0$ indican tendencia hacia el azul y $>0$ hacia el amarillo.

Para el $\mathrm{pH}$, se tomó $1 \mathrm{~mL}$ de jugo aproximadamente, se llevó a $50 \mathrm{~mL}$ con agua destilada, y se midió con un potenciómetro Hanna HI8424 (Hanna Instruments,Woonsocket, RI) previamente calibrado con soluciones buffer de $\mathrm{pH}$ 7,0 y 4,0. Los sólidos solubles totales (SST) se midieron a través de mediciones de grados ${ }^{\circ}$ Brix con un refractómetro digital Hanna HI 96803 (Hanna Instruments,Woonsocket, RI) de rango $0 \%$ a $85 \%$ con precisión $0,1^{\circ}$ Brix. Para la acidez total titulable (ATT), se tomó como base la metodología utilizada por Balaguera-López (2011) se realizaron cálculos con datos de volumen de $\mathrm{NaOH}$, incorporado en $1 \mathrm{~g}$ de jugo de frutos, llevado a $50 \mathrm{~mL}$ con agua destilada, agregando 5 gotas de fenoftaleína en una titulación potenciométrica hasta pH de 8,2. Para ello, se utilizó la fórmula:

$$
\% \text { Acidez }=\left(A^{*} B * C\right) * 100 / D
$$

Donde: $\mathrm{A}=$ volumen de $\mathrm{NaOH}$ gastado; $\mathrm{B}=$ Normalidad del $\mathrm{NaOH}(0,097)$; $\mathrm{C}=$ peso equivalente expresado en g de ácido predominante en el fruto (ácido cítrico 0,064 $\mathrm{g} \mathrm{meq}^{-1}$ ); D = masa en gramos de la muestra utilizada ( $5 \mathrm{~g})$.

La relación de madurez (RM) se calculó mediante la relación SST/ATT. Además se determinó la intensidad respiratoria (IR; $\mu \mathrm{L} \mathrm{CO} \mathrm{kg}^{-1} \mathrm{~h}^{-1}$ ), tomando aproximadamente $400 \mathrm{~g}$ de frutos de ciruela los cuales fueron colocados en cámaras herméticas VER BC-2000 (Vernier Software \& Technology, Beaverton, OR) de $250 \mathrm{~cm}^{3}$ durante 5 minutos, y luego se determinó la cantidad de $\mathrm{CO}_{2}$ mediante el equipo sensor VER $\mathrm{CO}_{2}$-BTA y el sistema de interfase LabQuest2 (Vernier Software \& Technology, Beaverton, OR).

Con los datos obtenidos se realizaron pruebas de normalidad (Shapiro-Wilk) y homocedasticidad de varianzas (test de Levene), análisis de varianza y pruebas de comparación de Tukey $(P \leq 0,05)$. Los análisis se realizaron con el programa estadístico SAS v. 9.2e (SAS Institute Inc., Cary, NC).

\section{RESULTADOS Y DISCUSIÓN}

\section{Pérdida de masa fresca}

La pérdida de masa fresca aumentó considerablemente durante el periodo de almacenamiento, aunque no hubo diferencia significativa entre estados de madurez en ninguna de las mediciones (figura 1A), situación muy similar a lo reportado por Sánchez y Saavedra (2013) en ciruela 'Horvin' en cinco estados de madurez y almacenada a temperatura ambiente. A los 9 días de almacenamiento (dda) se observó que en los estados 2 y 3 fue donde hubo mayor pérdida de masa fresca con valores de $12,27 \%$ y $11,90 \%$, en comparación con los estados 4 y 5 donde la pérdida acumulada final fue de $9,86 \%$ y $10,54 \%$ respectivamente. La mayor pérdida de masa obedece a la transpiración por efecto de la transferencia de masa en función del gradiente de humedad, área del fruto, temperatura y la humedad relativa, en la medida en que el producto se aproxima a la muerte tisular 
disminuye el gradiente de humedad y por ende la tasa de transpiración también (Mejia, 2013).

Parra-Coronado et al. (2008) encontraron que en frutos de ciruelo (Prunus salicina L.) variedad Horvin, almacenados a $4^{\circ} \mathrm{C}$ y $85 \%$ de humedad relativa, hasta el día 22 , el fruto ya no presentó características adecuadas para su comercialización, con una pérdida acumulada de masa fresca del $10,07 \%$, en nuestro caso la pérdida de peso fue mayor probablemente debido a la influencia de la aplicación de etileno exógeno, la cual conllevo a la disminución de la calidad de los frutos de ciruela (Wills y Warton, 2004), causada por la degradación de la pared celular, la cual acelera los procesos fisiológicos de transpiración y respiración lo que ocasiona mayores pérdidas de agua y sustratos (Kader, 2002).

En frutos de ciruela mexicana (Spondias purpurea L.) se observaron diferencias estadísticas a partir del cuarto día de almacenamiento a temperatura ambiente, en los tres estados de madurez (verde: frutos color verde intenso en toda la superficie; $1 / 2$ amarillo equivale a $50 \%$ de la superficie del fruto con color amarillo rojizo, y $3 / 4$ amarillo a $75 \%$ de la superficie del fruto con coloración amarillo rojizo) y condiciones de almacenamiento de $20 \pm 2^{\circ} \mathrm{C}$, con evaluación a los 0,2 y $4 \mathrm{~d}$; $8 \pm 2^{\circ} \mathrm{C}$ con $75-80 \%$ HR durante 1 semana, con evaluación a los 0,2 y 4 d, a temperatura ambiente; y $12 \pm 2{ }^{\circ} \mathrm{C}$ con $75-80 \%$ HR durante 1 semana, con evaluación 0,2 y $4 \mathrm{~d}$, a temperatura ambiente. Los síntomas de marchitamiento en los frutos, como la pérdida de humedad, fueron visibles cuando las pérdidas fueron superiores a $11 \%$ del peso inicial. En general, los frutos de los tres estados de madurez expuestos directamente a $20^{\circ} \mathrm{C}$ por $2 \mathrm{~d}$, perdieron entre 7,6 y $8,8 \%$ de su peso (Pérez- López et al., 2004).

\section{Firmeza}

La firmeza en los frutos de ciruela tratados con etileno presentó diferencias altamente significativas durante las dos primeras mediciones $(0$ y 3 d de almacenamiento), ya que existió una marcada diferenciación entre los estados de madurez de los frutos. A partir de los $6 \mathrm{~d}$ de almacenamiento no hubo diferencias significativas entre estados de madurez lo que demuestra que el etileno exógeno acelera la maduración de los frutos, lo cual produce características muy similares entre los frutos cosechados en estados inmaduros (2 y 3) y estados más avanzados (4 y 5) (figura 1B). Este efecto puede explicarse debido posiblemente a que una vez el fruto climatérico inicia el proceso de maduración, la concentración interna de etileno aumenta rápidamente y la posterior aplicación de etileno exógeno no causa efecto alguno en dicho proceso (Saltveit, 1999; Candan et al., 2008), lo que indica que en frutos en estado de madurez avanzado (4 y 5), el efecto del etileno exógeno es mínimo y por tanto no hay diferencia significativa con los frutos de estados inmaduros ( 2 y 3 ), donde el efecto es más notorio, lo cual coincide con lo reportado por Rincón et al. (2012) y Zapata et al. (2010) en arándanos en tres estados de madurez en donde no hubo cambios significativos en la firmeza.

Se observó que en los estados de madurez 2, 3 y 4 la firmeza de los frutos era superior al rango aceptable por el consumidor al momento de la cosecha, alrededor de 40 a $50 \mathrm{~N}$, pero al transcurrir 3 y 6 días, por efecto del etileno, ya se obtienen los valores de firmeza adecuados para el consumo, entre 10 y $20 \mathrm{~N}$ (Crisosto et al., 2004), lo que indica que los frutos pueden ser cosechados en estados tempranos para facilitar el transporte y disminuir el deterioro de los mismos y posteriormente en el punto de venta realizar el tratamiento con etileno, para obtener rápidamente la maduración de consumo.

\section{Índice de color}

El índice de color (IC; figura 2D) y los valores de $L^{*}, a^{*}$ y $b^{*}$ en frutos de ciruela presentaron diferencias significativas en la mayoría de los puntos de medición, lo que permite observar la aceleración en el cambio de color provocada 


\section{A}

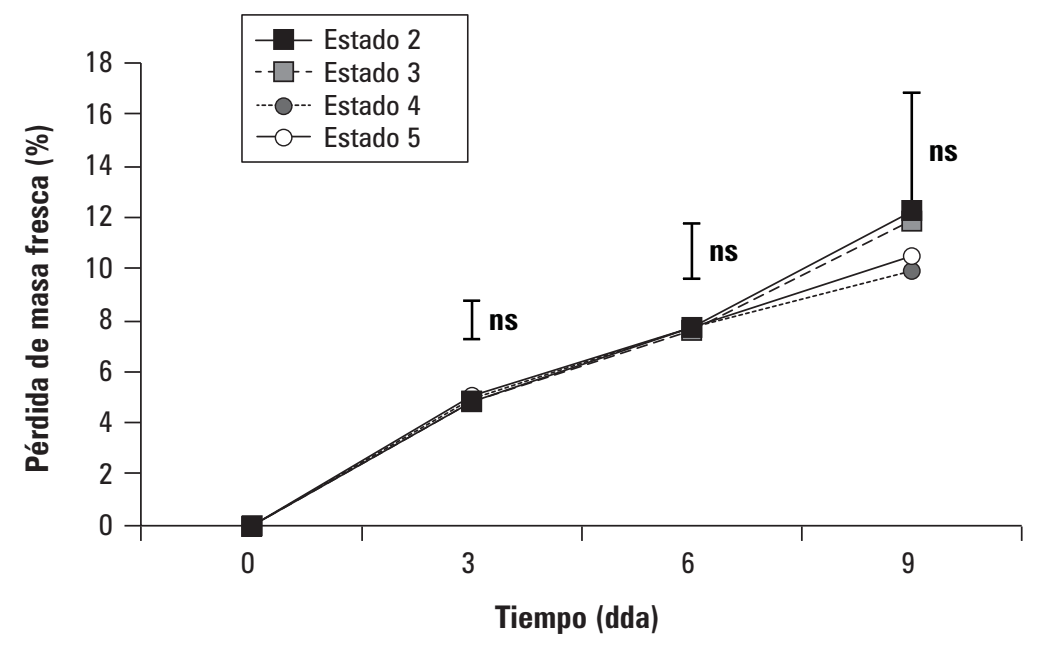

B

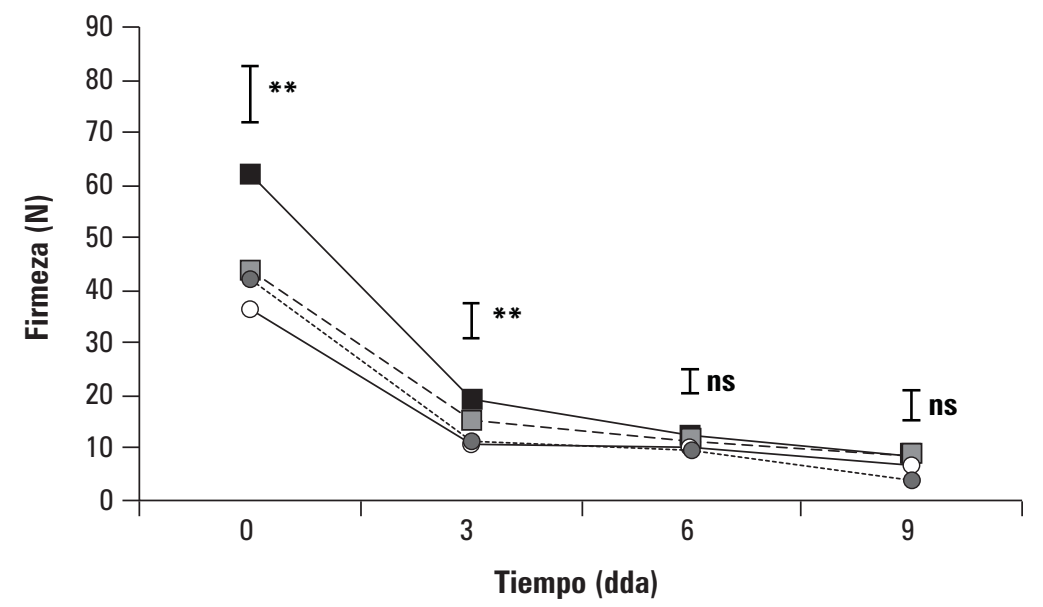

Figura 1. Efecto de la aplicación de etileno (100 $\left.\mu \mathrm{L} \mathrm{L}^{-1}\right)$ sobre A) la pérdida de masa fresca y B) la firmeza en frutos de ciruela 'Horvin' durante la poscosecha. La barra indica la diferencia mínima significativa según la prueba de Tukey $(P \leq 0,05)$. Si las diferencias entre dos promedios en cada punto de muestreo son mayores a la prueba de Tukey, entonces habrá diferencia a un $\alpha$ de 0,05; * diferencias estadísticas al 5\%; ** diferencias estadísticas al $1 \%$; ns no hay diferencias.

por la aplicación de etileno. En todos los estados de madurez, los frutos al finalizar el periodo de almacenamiento presentaron un color rojo brillante pero de diferente intensidad. Analizando cada uno de los valores individualmente, el valor de $\mathrm{L}$ en los frutos siempre fue mayor en los estados 2 y 3 , como se observa en la figura $2 \mathrm{~A}$, ya que en estos estados el color fue más claro que en los estados más maduros (4 y 5), donde el rojo es más oscuro pero menos brillante, aunque al final del periodo de almacenamiento se presentó un aumento de este valor en todos los estados. Un comportamiento similar se observó en el valor $b^{*}$, en donde los valores en la primera medición fueron altos en todos los estados, a los 3 y $6 \mathrm{~d}$ disminuyeron considerablemente y al cabo de los 9 dda volvieron a incrementarse, como se observa en la figura 2C, lo que indica 
que los frutos pierden color azul y pasan a ganar una mayor tonalidad amarilla. El valor $a^{*}$ mostró diferencias altamente significativas en la mayoría de las mediciones (figura 2B). Los estados en donde el cambio fue más radical fueron el 2, con un valor inicial de -8,27, el cual cambió a 26,96 a los 3 dda, y el 3 con un valor inicial de 3,99 y a los 3 dda de 21,81. En los estados 4 y 5 se observó un leve cambio. Este cambio de color se produce debido tanto a la maduración normal del fruto como a la aceleración causada por la aplicación exógena de etileno (Osuna et al., 2005) como sucede en las ciruelas japonesas (Khan y Singh, 2008), esto debido a la degradación de la clorofila y el consecuente incremento de la concentración de antocianinas (Ashton et al., 2006).

\section{$\mathrm{pH}$}

El pH no presentó diferencias significativas entre estados de madurez, y en general hubo muy poca variación entre los valores obtenidos al inicio del estudio $(3,07)$ y los obtenidos al finalizar $(3,48)$ para el estado 2 . De forma similar ocurrió para el estado 5 , ya que este comenzó con un valor de 3,52 y finalizó con 3,96 a los 9 dda, lo que indica que la aplicación de etileno tiene muy poco efecto sobre el cambio de $\mathrm{pH}$ en los frutos de ciruela (figura 3A). Al respecto, Sánchez y Saavedra (2013) encontraron diferencias significativas entre los valores de $\mathrm{pH}$ obtenidos en frutos de ciruela en cinco estados de madurez; pero al inicio y al final del almacenamiento (29 días), no hubo diferencia significativa entre estados de madurez, además la variación entre los datos iniciales y finales en cada estado mostró un leve aumento como ocurrió en el presente estudio. No obstante, en ciruelas Prunus persica, cv. Rich Lady y Prunus salicina, cv. Black Splendor y Prunus persica, cv. Andros, durante la etapa de maduración y sobremaduración no hubo cambios en el pH ni en el contenido de SST durante 7 dda (Puerta-Gómez y CisnerosZevallos, 2011; Manganaris et al., 2007).

\section{Acidez total titulable (ATT)}

Esta variable presentó diferencias significativas solamente al inicio del estudio y a los 3 dda, mientras que a los 6 y 9 dda no hubo desemejanzas significativas, lo que indica que las diferencias presentadas al comienzo se deben seguramente a la diferencia entre los estados de madurez de los frutos en la cosecha y conforme transcurre el tiempo del almacenamiento, la aplicación de etileno acelera la maduración de los frutos disminuyendo las diferencias. La ATT en los frutos de ciruela disminuyó a los $6 \mathrm{dda}$, efecto que fue independiente del estado de madurez en que fueron cosechados los frutos, lo cual es un comportamiento típico de la maduración, en donde se presenta una disminución en la ATT y un aumento de los SST (Díaz-Mula et al., 2011). El porcentaje de disminución de la ATT fue mayor en los frutos cosechados en estado 2 , obteniendo un $61,95 \%$, seguido del estado 3,5 y 4 con valores de $51,23 \%, 41,83 \%$ y $32 \%$, lo que indica un mayor efecto de la aplicación del etileno exógeno en los estados inmaduros, acelerando la maduración y consiguiendo valores muy similares a los estados 4 y 5 . Esta disminución de la acidez se relaciona con el proceso de maduración, ya que los ácidos son sustratos respiratorios o son convertidos a azúcares (Balaguera-López, 2011).

A los $9 \mathrm{~d}$ se produjo un aumento en la ATT en todos los estados (figura 3B), debido posiblemente a un aumento en la actividad de la enzima citrato sintasa (CS), la cual puede ser inducida en los primeros estados de desarrollo (Balaguera-López, 2011). Por lo tanto, se puede inferir que la aplicación de etileno exógeno aumentó la concentración de azúcares solubles y provocó la disminución del contenido de ácidos orgánicos y compuestos fenólicos (Kays, 2004). Además se puede establecer que para ciruela Horvin cosechada en diferentes estados de madurez, la aplicación de etileno provoca la disminución de la acidez en los frutos, sin diferencias estadísticas entre los estados de madurez, lo que sugiere que aun cuando los frutos hayan sido cosechados en un estado inmaduro, con la aplicación de etileno es posible lograr rápidamente una calidad homogénea del fruto. 


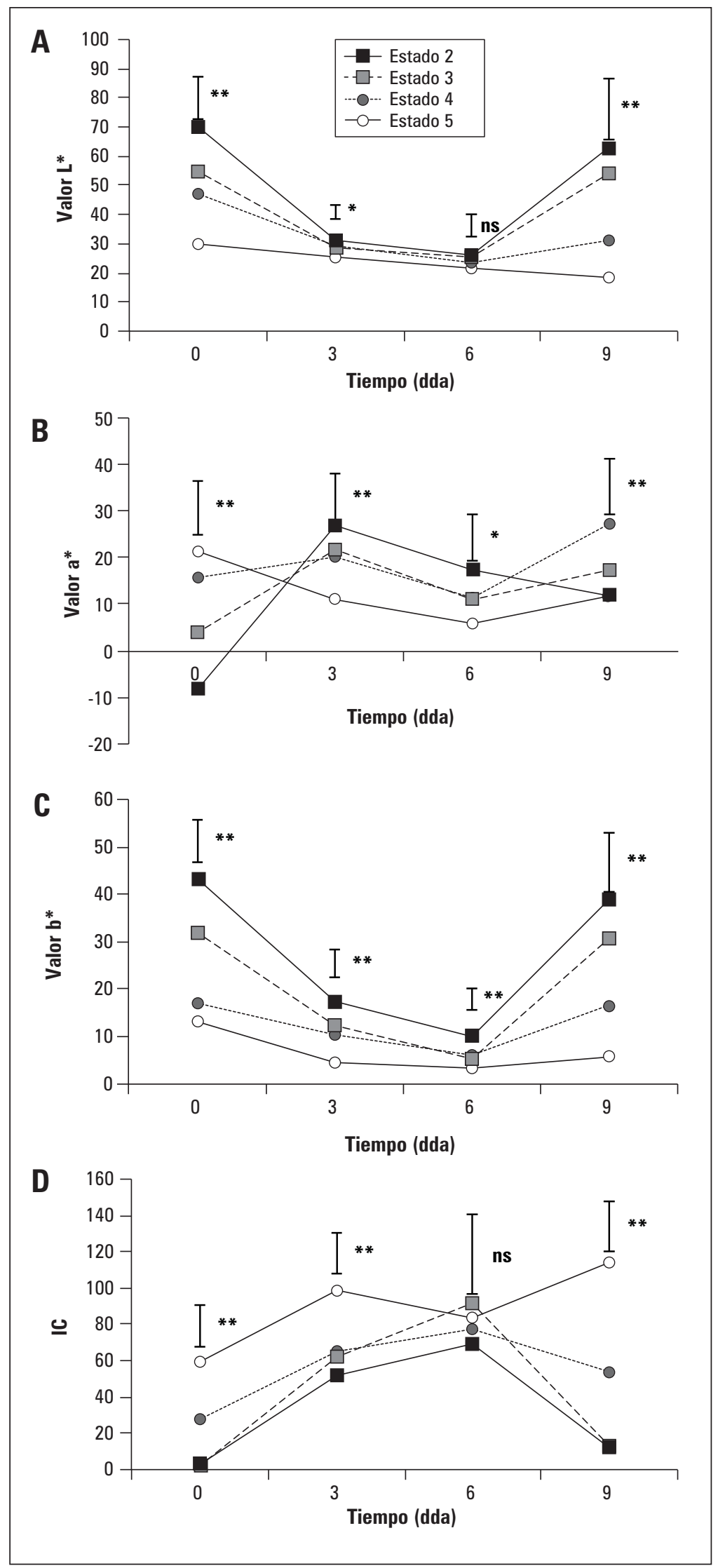

Figura 2.

Efecto del etileno $\left(100 \mu L^{-1}\right)$ sobre $A$ ) valor de L (luminosidad), B) valor de $\mathrm{a}^{*}$ (cromaticidad de verde a rojo), C) valor de $b^{*}$ (cromaticidad de azul a amarillo) y D) índice de color (IC) en frutos de ciruela 'Horvin' en cuatro estados de madurez durante la poscosecha. La barra indica la diferencia mínima significativa según la prueba de Tukey $(P \leq 0,05)$. Si las diferencias entre dos promedios en cada punto de muestreo son mayores a la prueba de Tukey, entonces habrá diferencia a un $\alpha$ de 0,$05 ;$ * diferencias estadísticas al 5\%; ** diferencias estadísticas al $1 \%$; ns no hay diferencias. 
A

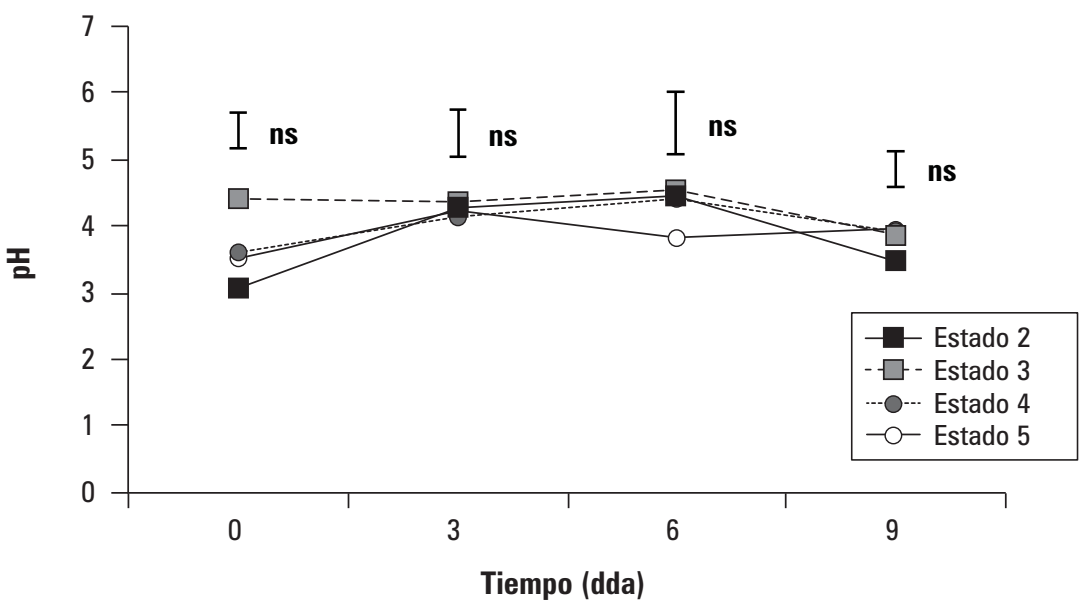

B

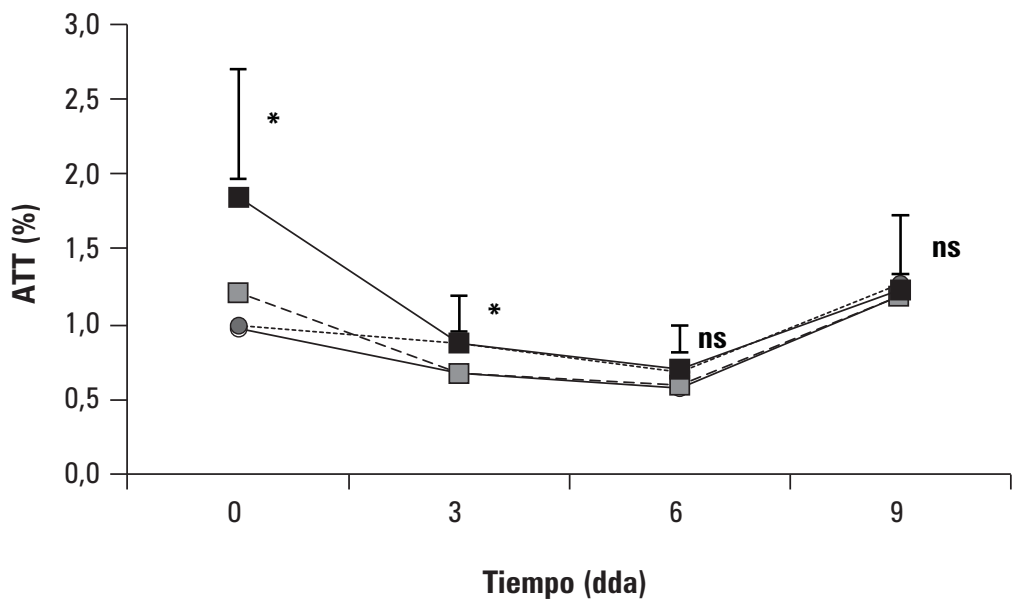

Figura 3. Efecto de la aplicación de etileno $\left(100 \mu \mathrm{L} \mathrm{L}^{-1}\right)$ sobre el A) pH y B) acidez total titulable (ATT) en frutos de ciruela 'Horvin' en cuatro estados de madurez durante la poscosecha. La barra indica la diferencia mínima significativa según la prueba de Tukey $(P \leq 0,05)$. Si las diferencias entre dos promedios en cada punto de muestreo son mayores a la prueba de Tukey, entonces habrá diferencia a un $\alpha$ de 0,05; * diferencias estadísticas al $5 \%$; ** diferencias estadísticas al $1 \%$; ns no hay diferencias.

\section{Sólidos solubles totales (SST)}

No se presentaron diferencias significativas en la mayoría de los puntos de medición, lo que indica que la aplicación de etileno no afectó los SST presentes en los frutos cosechados en diferente estado de madurez (figura 4a) y tampoco generó un aumento significativo de los SST desde el inicio del almacenamiento y hasta el final del mismo (9 dda), lo cual coincide con Rozo-Romero et al. (2015) quienes aplicaron en ciruela japonesa concentraciones de etileno entre 0 y $1.000 \mathrm{mg}$ $\mathrm{L}^{-1}$ sin un efecto significativo sobre los SST. Por otro lado, Infante et al. (2011) reportaron que en ciruelas japonesas almacenadas durante $10 \mathrm{~d}$ a $22^{\circ} \mathrm{C}$, los SST tuvieron un aumento poco significativo, lo que indica que este parámetro, aunque está asociado con el sabor y la calidad de los frutos, no podría ayudar a determinar el punto de cosecha de forma precisa (Crisosto et al., 2004). 

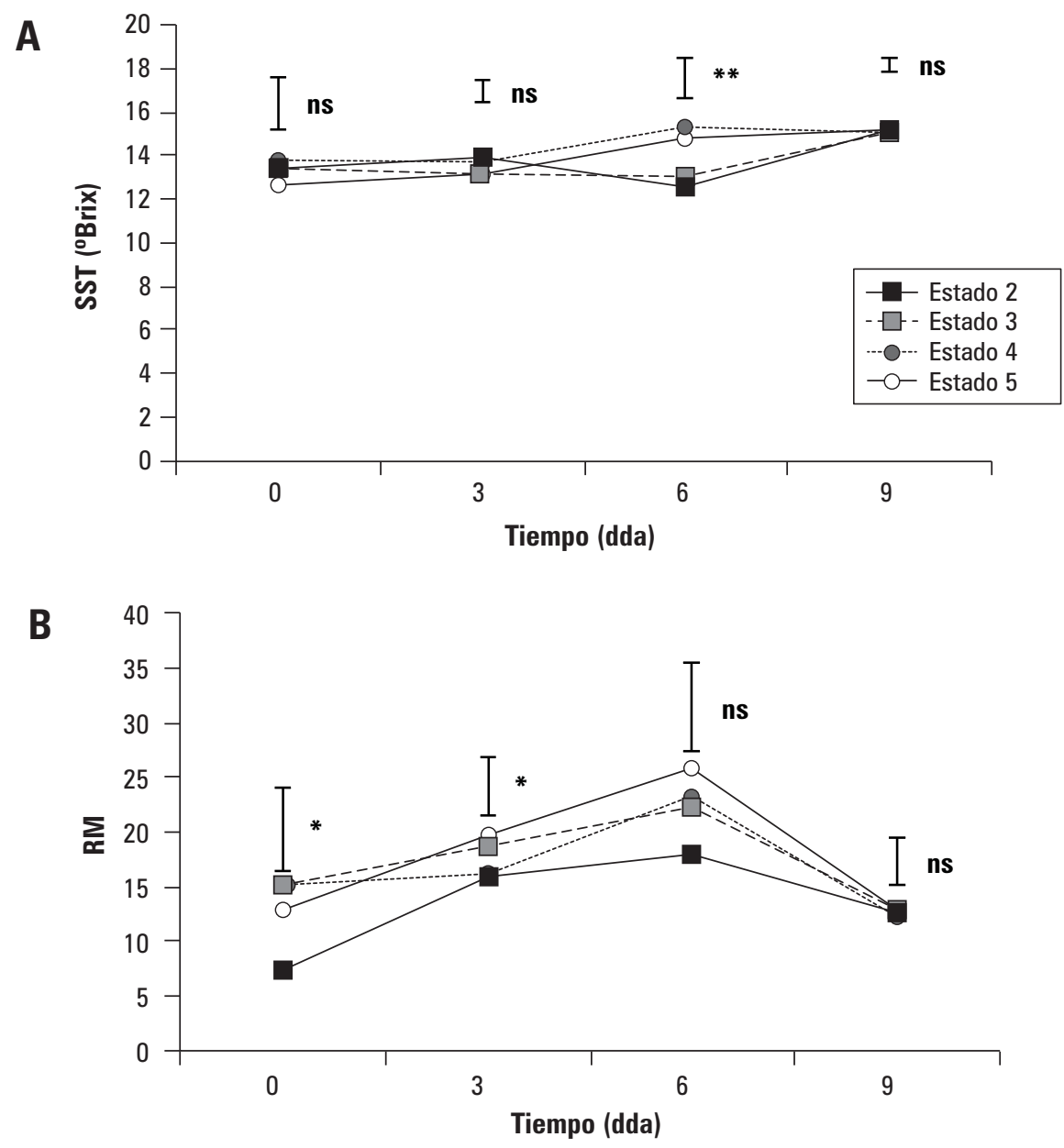

Figura 4. Efecto de la aplicación de etileno (100 $\mu$ L L-1) sobre A) sólidos solubles totales (SST) y B) relación de madurez (RM; SST/ATT) en frutos de ciruela 'Horvin' en cuatro estados de madurez durante la poscosecha. La barra indica la diferencia mínima significativa según la prueba de Tukey $(P \leq 0,05)$. Si las diferencias entre dos promedios en cada punto de muestreo son mayores a la prueba de Tukey, entonces habrá diferencia a un $\alpha$ de 0,05; * diferencias estadísticas al 5\%; ** diferencias estadísticas al 1\%; ns no hay diferencias.

\section{Relación de madurez (RM)}

En los frutos de ciruela solo se observaron diferencias significativas al inicio y a los 3 dda en la RM, lo que indica que la RM al transcurrir el tiempo es similar en los frutos cosechados inmaduros $(2$ y 3$)$ y los cosechados en estados más avanzados (4 y 5). Lo anterior permite inferir que la aplicación exógena de etileno logra acelerar la maduración de los frutos, independientemente del estado en que sean cosechados; no obstante al terminar el periodo poscosecha, la RM descendió en todos los estados, sin encontrarse diferencia significativa entre ellos, lo que indica el inicio de la senescencia.

Los frutos cosechados en todos los estados de madurez presentaron los mayores valores de $\mathrm{RM}$ a los $6 \mathrm{dda}$, lo que sugiere que para frutos de ciruela tratados con etileno, este es el tiempo máximo en el cual los frutos se pueden comercializar pues este índice sirve para medir la calidad organolép- 
tica de los frutos (Rodríguez et al., 2006), ya que como se observa en la figura $4 \mathrm{~B}$, esta relación a partir del día seis comienza a disminuir. Según Kays (2004), el aumento de la RM ocurre cuando los frutos alcanzan la máxima intensidad respiratoria, del mismo modo, Guillén et al. (2013) afirman que los frutos al estar expuestos a la acción normal del etileno maduran más rápidamente.

\section{Intensidad respiratoria (IR)}

La IR $\left(\mu \mathrm{L} \mathrm{CO}_{2} \mathrm{~kg}^{-1} \mathrm{~h}^{-1}\right)$ mostró diferencias significativas en todos los puntos de muestreo, excepto en el último. Se observó un aumento constante de la IR hasta los 6 dda y luego disminuyó (figura 5). El aumento en la IR en los frutos es un proceso normal, que implica una serie de reacciones de óxido-reducción en las cuales diversas sustancias dentro de las células se oxidan a $\mathrm{CO}_{2}$ para generar ATP y esqueletos de carbono para la síntesis de compuestos durante la maduración (Bhande et al., 2008). El pico climatérico en los frutos evaluados se produjo a los 6 dda en la mayoría de los tratamientos, por lo que la aplicación de etileno aumentó el valor de la IR pero no adelantó la llegada del pico climatérico, similar a lo encontrado en ciruelas por Singh y Singh (2012). Por el contrario, el uso de retardantes de madurez y revestimientos hace que disminuya la producción de etileno y los valores de la IR (Guillén et al., 2013), lo cual puede ser atribuido a la modificación de la atmósfera interna (aumento de $\mathrm{CO}_{2}$ y la disminución de $\mathrm{O}_{2}$ ) que reduce potencialmente la producción de etileno (Valero et al., 2013), con lo cual se confirma la acción del etileno sobre la aceleración de la madurez. Lo anterior concuerda con lo reportado por MontalvoGonzález et al. (2009) y Mayuoni et al. (2011) quienes observaron en frutos de chile poblano, la aplicación de etileno exógeno redujo un efecto importante en la actividad enzimática del ciclo respiratorio, regulando la expresión genética y aumentando la actividad metabólica, al igual que la tasa de producción de etileno endógeno.

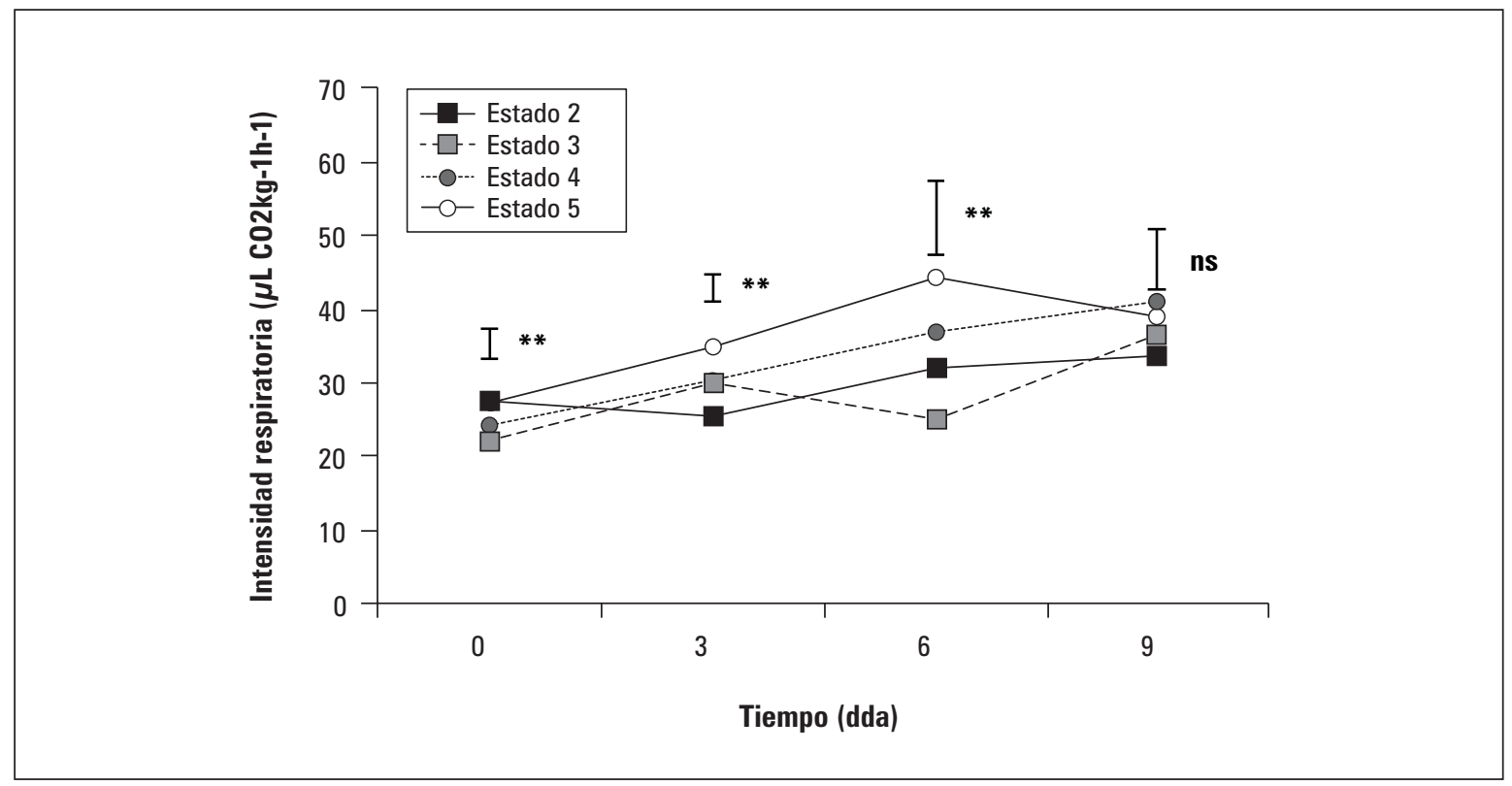

Figura 5. Efecto de la aplicación de etileno $\left(100 \mu \mathrm{L} \mathrm{L}^{-1}\right)$ sobre la tasa respiratoria en frutos de ciruela 'Horvin' en cuatro estados de madurez durante la poscosecha. La barra indica la diferencia mínima significativa según la prueba de Tukey $(P \leq 0,05)$. Si las diferencias entre dos promedios en cada punto de muestreo son mayores a la prueba de Tukey, entonces habrá diferencia a un $\alpha$ de 0,05; * diferencias estadísticas al $5 \%$; ** diferencias estadísticas al $1 \%$; ns no hay diferencias. 


\section{CONCLUSIONES}

El estado de madurez en que los frutos son cosechados tiene gran incidencia durante la poscosecha debido a que los frutos cosechados en estado 2 mostraron un mayor porcentaje de pérdida de masa y la mayor ATT, los frutos en estado 5 de madurez fueron los que presentaron mayores valores en SST, pH, IC, RM e IR, al igual que mayor pérdida de firmeza.

La aplicación de etileno en frutos de ciruela acelera el proceso de maduración independien- temente del estado de madurez en el cual se han cosechado. El estado de madurez en el que se observó mayor efectividad en la aplicación de etileno es el estado 3, denotándose un mayor cambio de color y la pérdida de masa es menor que en el estado 2. La RM es acelerada por la aplicación de etileno en todos los estados, pero en los estados 2 y 3 es donde se conserva al final del estudio una firmeza aceptable por el consumidor. La aplicación de etileno incrementó la IR en todos los estados, favoreciendo la maduración sin adelantar el pico climatérico.

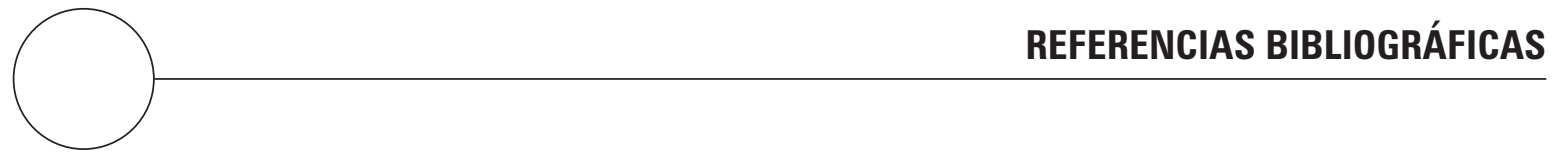

Abdi, N., W.B. McGlasson, P. Holford, M. Williams y Y. Mizrahi. 1998. Responses of climateric and suppressed-climateric plums to treatment with propylene and 1-methylcyclopropene. Postharvest Biol. Technol. 14, 29-39. Doi: 10.1016/s09255214(98)00031-3

Ashton, O.B.O., M. Wong, T.K. Mcghie, R. Vather, Y. Wang, C. Requejo, P. Ramankutty y A.B. Woof. 2006. Pigments in avocado tissue and oil. J. Agr. Food Chem. 54, 10151-10158. Doi: 10.1021/ jf061809j

Balaguera-López, H. 2011. Estudio del crecimiento y desarrollo del fruto de champa (Campomanesia lineatifolia $\mathrm{R} \& \mathrm{P}$ ) y determinación del punto óptimo de cosecha. Tesis de maestría. Facultad de Agronomía, Universidad Nacional de Colombia, Bogotá.

Bhande S. D., M.R. Ravindra y T.K. Goswami. 2008. Respiration rate of banana fruit under aerobic conditions at different storage temperatures. J. Food Eng. 87(1), 116-123. Doi: 10.1016/j.jfoodeng.2007.11.019

Candan, A.P., J. Graell y C. Larrigaudiere. 2008. Roles of climacteric ethylene in the development of chilling injury in plums. Postharvest Biol. Technol. 47, 107112. Doi: $10.1016 /$ j.postharvbio.2007.06.009

Crisosto, C.H., D. Garner, G.M. Crisosto y E. Bowerman. 2004. Increasing 'Black amber' plum (Prunus salicina Lindl.) consumer acceptance. Postharvest
Biol. Technol. 34, 237-244. Doi: 10.1016/j.postharvbio. 2004.06 .003

Deaquiz, Y., J. Álvarez-Herrera y G. Fischer, 2014. Ethylene and 1-MCP affect the postharvest behavior of yellow pitahaya fruits (Selenicereus megalanthus Haw.). Agron. Colomb. 32(1), 44-51. Doi: 10.15446/agron.colomb.v32n1.41950

De la Cruz, J., G. Vela, L. Dorantes y H.S. García. 2009. Efecto del etileno sobre el ACC y ACC oxidasa en la maduración de papaya 'Maradol'. Rev. Fitotec. Mex. 33(2), 133-140.

Díaz-Mula, H.M., P.J. Zapata, F. Guillén, J.M. Valverde, D. Valero y M. Serrano. 2011. Modified atmosphere packaging of yellow and purple plum cultivars. 2. Effect on bioactive compounds and antioxidant activity. Postharvest Biol. Technol. 61, 110-116. Doi: 10.1016/j.postharvbio.2011.02.012

Guillén, F., H. Díaz-Mula, P. Zapata, D. Valero, M. Serrano, S. Castillo y D. Martínez-Romero. 2013. Aloe arborescens and Aloe vera gels as coatings in delaying postharvest ripening in peach and plum fruit. Postharvest Biol. Technol. 83, 54-57. Doi: 10.1016/j.postharvbio.2013.03.011

Hancock, J., P. Callow, S. Sercxe, E. Hanson y R. Beaudry. 2008. Effect of cultivar, controlled atmosphere storage, and fruit ripeness on the longterm storage of highbush blueberries. Hort Technol. 18(2), 199-205. 
Infante, R., L. Contador, P. Rubio, K. Mesa y C. Meneses. 2011. Non-destructive monitoring of flesh softening in the black-skined Japanese plums 'Angeleno' and 'Autumn beaut' on-tree and postharvest. Postharvest Biol. Technol. 61, 35-40. Doi: 10.1016/j.postharvbio.2011.01.003

Kader, A. (ed.). 2002. Postharvest technology of horticultural crops. $3^{\text {rd }}$ ed. Division of Agriculture and Natural Resources, University of California, Oakland, CA.

Kays, S. 2004. Postharvest biology. Exon Press, Athens, GA.

Khan, A.S. y Z. Singh. 2008. 1-Methylcyclopropene application and modified atmosphere packaging affect ethylene biosynthesis, fruit softening, and quality of 'Tegan Blue' Japanese plum during cold storage. J. Amer. Soc. Hort. Sci. 133, 290-299.

Manganaris, G.A., M. Vasilakakis, G. Diamantidis y I. Mignani. 2007. The effect of postharvest calcium application on tissue calcium concentration, quality attributes, incidence of flesh browning and cell wall physicochemical aspects of peach fruits. Food Chem. 4, 1385-1392. Doi: 10.1016/j.foodchem.2005.11.036

Mayuoni L., Z. Tietel, B. Patil y R. Porat. 2011. Does ethylene degreening affect internal quality of citrus fruit? Postharvest Biol. Technol. 62, 50-58. Doi: 10.1016/j.postharvbio.2011.04.005

Mejía, L. 2013. Evaluación del comportamiento físico y químico poscosecha del plátano dominico hartón (Musa aab simmonds) cultivado en el municipio de Belalcázar (Caldas). Tesis de maestría. Facultad de Ciencias, Universidad Nacional de Colombia, Bogotá.

Montalvo-González, E., N. González-Espinoza, H. García-Galindo, B. Tovar-Gómez y M. Mata-Montes de Oca, 2009. Efecto del etileno exógeno sobre la desverdización del chile 'poblano' en poscosecha. Rev. Chapingo Ser. Hortic. 15(2), 189-197.

Osuna-García J.A., J.A. Beltrán y M.H. Pérez-Barraza. 2005. Mejoramiento de la vida de anaquel y calidad de papaya 'Maradol' con el 1-Metilciclopropeno (1-MCP). Rev. Chapingo Ser. Hortic. 11(1), 7-12.

Paliyath, G. y D. Murr. 2008. Biochemistry of fruits. pp. 19-50. En: Paliyath, G., D. Murr, A. Handa y S. Lurie (eds.). Postharvest biology and tecnology of fruits, vegetables and flowers. Wiley-Blackwell Publishing, Ames, IA.
Parra-Coronado, A., Hernández- Hernández, J y Camacho-Tamayo, J. 2008. Estudio fisiológico poscosecha y evaluación de la calidad de la ciruela variedad Horvin (Prunus domestica L.) bajo tres condiciones de almacenamiento refrigerado. Rev. Ing. Invest. 28(1), 99-104

Pérez-López, A., C. Saucedo, M. Arévalo y A. Muratalla 2004. Efecto del grado de madurez en la calidad y vida poscosecha de ciruela mexicana (Spondias purpurea L.). Rev. Fitotec. Mex. 27(2), 133- 139.

Puerta-Gómez, A. y L. Cisneros-Zevallos. 2011. Postharvest studies beyond fresh market eating quality: Phytochemical antioxidant changes in peach and plum fruit during ripening and advanced senescence. Postharvest Biol. Technol. 60, 220-224. Doi: 10.1016/j.postharvbio.2011.01.005

Reddy, N.S. y K. Haripriya. 2002. Extension of storage life of mango cvs. Bangalora and Neelum. South Indian Hortic. 50, 7-18.

Rincón, M.C., C.M. Buitrago, G.A. Ligarreto, W.S Torres y H.E. Balaguera. 2012. Comportamiento del fruto de Agraz (Vaccinium meridionale Swartz) cosechado en diferentes estados de madurez y almacenado en refrigeración. Rev. Fac. Nal. Agron. Medellín 65(2), 6615-6625.

Rodríguez, M., H. Arjona y J. Galvis. 2006. Maduración del fruto de feijoa (Acca sellowiana Berg) en los clones 41 (Quimba) y 8-4 a temperatura ambiente en condiciones de Bogotá. Agron. Colomb. 24(1), 6876.

Rozo-Romero, X., J.G. Álvarez-Herrera y H. Balaguera-López. 2015. Ethylene and changes during ripening in plum fruits Horvin (Prunus domestica L.). Agron. Colomb. 33(2). Doi: 10.15446/agron.colomb.v33n2.49856

Salcedo, A. 2014. Evaluación de absorbedores de etileno compuestos por mezclas de permanganato de potasio y vermiculita como retardantes de madurez de frutos de tomate (Solanum lycopersicum L.). Tesis de maestría. Facultad de Ciencias Agrarias, Universidad Nacional de Colombia, Bogotá.

Saltveit, M. 1999. Effect of ethylene on quality of fresh fruits and vegetables. Postharvest Biol. Technol. 15, 279-292. Doi: 10.1016/s0925-5214(98)00091-x

Sánchez, L. y G. Saavedra. 2013. Comportamiento poscosecha de frutos de ciruelo (Prunus domestica L.) var. Horvin cosechados en diferentes grados de madurez. Trabajo final. Programa de Especialización, Facultad de Ciencias Agropecuarias, Uni- 
versidad Pedagógica y Tecnológica de Colombia, Duitama, Colombia.

Singh, S.P. y Z. Singh. 2012. Postharvest oxidative behaviour of 1-methylcyclopropene treated japanese plums (Prunus salicina Lindl.) during storage under controlled and modified atmospheres. Postharvest Biol. Technol. 74, 26-35. Doi: 10.1016/j. postharvbio.2012.06.012

Tapia, F. y G. Reginato, 2012. Efecto de la época de cosecha sobre la productividad y calidad del fruto en ciruelo europeo variedad D'Agen. Antumapu Profesional 1(1), 1-7.

Valero, D., M. Díaz-Mula, P. Zapata, F. Guillén, D. Martínez-Romero, S. Castillo y M. Serrano. 2013. Effects of alginate edible coating on preser- ving fruit quality in four plum cultivars during postharvest storage. Postharvest Biol. Technol. 77, 1-6. Doi: /10.1016/j.postharvbio.2012.10.011

Verma, L.R. y V.K, Joshi. 2000. Postharvest technology of fruits and vegetables: an overview. Indus Publishing Co., New Delhi.

Wills, R.B.H. y M.A. Warton. 2004. Efficacy of potassium permanganate impregnated into alumina beads to reduce atmospheric ethylene. J. Amer. Soc. Hort. Sci. 129, 433-438.

Zapata, L.M., A.D. Malleret, C.F. Quinteros, C.E. Lesa, C.O. Vuarant, M.F. Rivadeneira y J.A. Gerard. 2010. Estudio sobre cambios de la firmeza de bayas de arándanos durante su maduración. Cienc. Docencia Tecnol. 21(41), 159-171. 